\title{
ON MATURATION IN THE EGGS OF LIMNAEA STAGNALIS L.
}

BY

\author{
CHR. P. RAVEN
}

The nuclear phenomena of maturation and fertilization in Limnaea stagnalis have been described by me (RAven 1945). Freshly-laid eggs contain the first maturation spindle in metaphase; it has a moderate aster at both ends (fig. Ia). The sperm head is situated immediately beneath the egg cortex as a compact dark body. At a late anaphase stage, the outer end of the maturation spindle comes into contact with the egg surface at the animal pole; at this place, a pit-like depression of the surface appears. The aster at this end of the spindle flattens against the surface (fig. Ib). Ten minutes later, the formation of the first polar body begins, as a conical or hemispherical projection at the animal pole. The astral rays at this end disappear more and more as the extrusion of the polar body progresses. The aster at the inner end of the maturation spindle, on the contrary, has grown very large; it contains a big clear "central body" (fig. Ic). At the time the dyads have reached the spindle poles the first polar body is pinched off. The number of dyads was determined as I6-18.

The spermaster makes its appearance shortly before extrusion of the first polar body. At first, it is a small structure lying near the centre of the egg, at some distance from the deep pole of the maturation spindle. It contains no "central body", the astral rays meeting in one point at the centre of the spermaster (fig. Ic). During the formation of the second maturation spindle, it is growing, and a small "central body" appears (fig. Id). Meanwhile, the sperm head retains its subcortical position.

The second maturation spindle is shorter than the first; the asters at its ends are at first rather inconspicuous (fig. Id); later, large asters appear, especially at the deep end of the spindle (fig. Ie). The extrusion of the second polar body is accompanied with the same phenomena as that of the first. A spindle-remnant and associated "mid-body" may be found until one hour after its formation (fig. If, g).

Directly after the completion of the second maturation division, the chromosomes of the egg nucleus swell into little vesicles, containing each a dark 
granule; 18 of such karyomeres could be counted. They are lying at first in the inner aster of the maturation spindle; later, they are found in the subcortical protoplasm at the animal pole of the egg (fig. Ig).

The spermaster at the time of second polar body formation has attained a stage, in which a central area of clear, highly vacuolated protoplasm is surrounded by a zone of denser proloplasm, in which the astral rays are
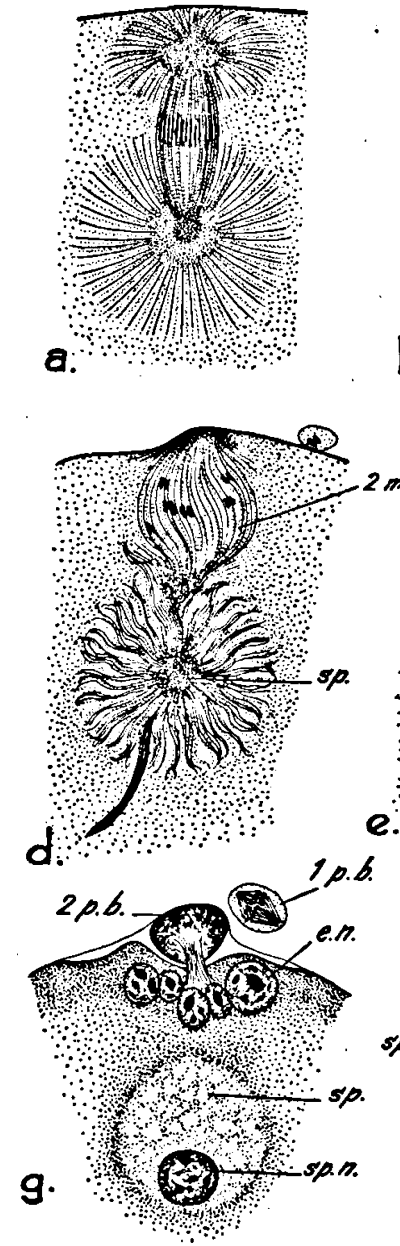

b.
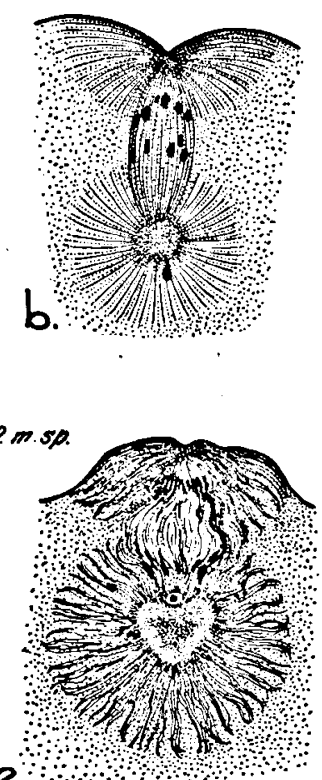
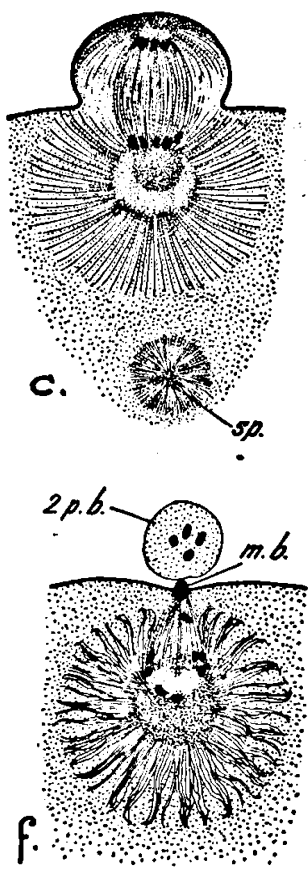

h.
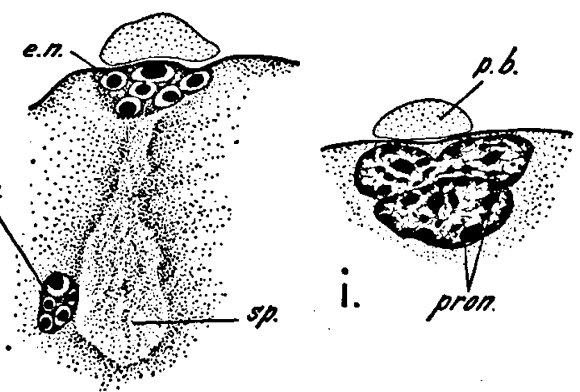

Fig. I. Maturation and fertilization in Limnaca stagnalis. Eggs in eggcapsule fluid. $a$. Ist maturation spindle, early anaphase. $b$. Late anaphase. Spindle end in contact with egg cortex. $c$. Telophase. Formation of ist polar body. Spermaster (sp.) just formed. $d$. Formation of $2 \mathrm{~d}$ maturation spindle (2. m. sp.). Spermaster, in connexion with sperm: tail, near deep end of spindle. $e$. Late anaphase of $2 \mathrm{~d}$ maturation division. f. $2 \mathrm{~d}$ polar body (2. p. b.) formed. Spindle remnant and mid-body (m.b.). $g$. Karyomeres of egg nucleus (e.n.) in subcortical plasm. Sperm nucleus (sp. n.) in central area of spermaster (sp.). $h$. Sperm nucleus beside spermaster. $i$. Copulation of pronuclei (pron.). 
no longer very conspicuous (fig. Ig). Shortly after the extrusion of the second polar body the sperm head, which has remained inactive till this moment, leaves its subcortical position and moves to the spermaster, where it comes to lie in the central clear area (fig. Ig). At the same time, it develops into the male pronucleus. In the second hour after the completion of maturation, the spermaster shifts towards the animal pole of the egg; likewise the male pronucleus moves to the animal pole, where it meets the female pronucleus (fig. Ii). The pronuclei have a rather irregular outline at first. The spermaster vanishes altogether. About 20 minutes later, the nuclear membranes are lost and the cleavage spindle makes its appearance.

In connection with our experiments on the influence of lithium chloride upon the uncleaved eggs of Limnaea, it proved necessary to study more accurately some details of the maturation processes in normal eggs. Thereby some interesting facts were discovered adding to our knowledge of the nuclear phenomena of maturation and fertilization in Limnaea.

\section{MATERIAL}

It appeared that the accurate details of the intricate processes occurring in the egg could only be elucidated by means of an extensive material. For the study of the period lying between the telophase of the first and the end of the second maturation division, no less than 125 eggs have been used. Part of them had developed in the egg-capsules, the other had been decapsulated immediately after oviposition and had developed in distilled water. No differences in the course of the maturation divisions between both groups of eggs could be observed; apparently, these processes continue in an entirely normal way in distilled water. The eggs had been fixed in Bouin's fluid, sectioned at $5 \mu$ and stained with iron haematoxylin and saffranin or with azan. Cameralucida drawings of the nuclei, spindles and asters of all eggs have been made. From these drawings, the course of the processes could be reconstructed step by step.

\section{RESULTS}

As regands the first phases of the maturation process, nothing has to be added to our former description. At the end of the anaphase of the first maturation division, the outer aster, which is situated in the conical to hemispherical rudiment of the first polar body, is considerably reduced; the inner aster is big and has a clear central area ("central body") (fig. 2a). Within this area, no centriole ever has been found; such a body does not exist at all or it cannot be demonstrated with any of the methods of fixation and staining used in Limnaea stagnalis eggs up till now. I wish to emphasize, furthermore, that the central area of the asters (so-called "centrosome" of some authors, "centrosphere" of other ones-the terminology relating to these 
structures is rather confusing !-) is no "body" in the proper sense of the word, but an area of protoplasm which differs from the surrounding region of astral rays in its submicroscopic structure; presumabiy, the ondered radial arrangement of the cytoplasmic micelles has given way here to a less regular,

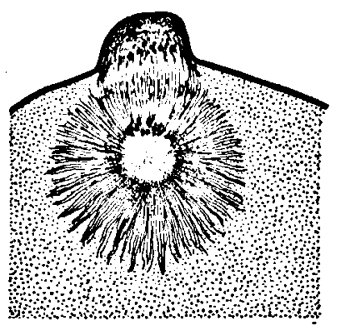

a.

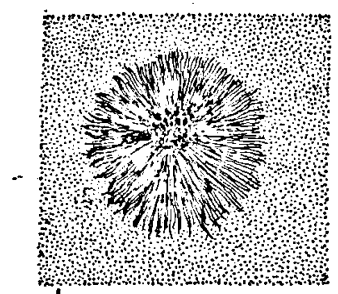

d.
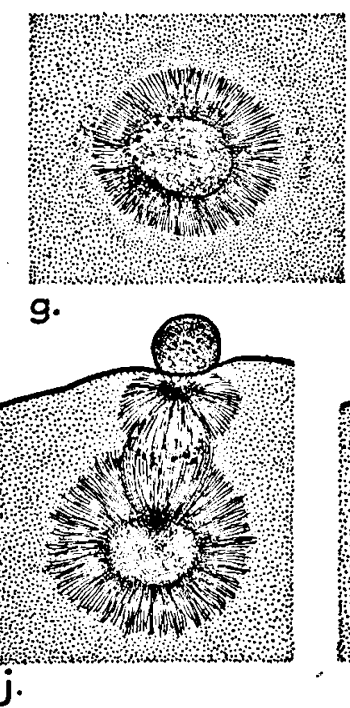

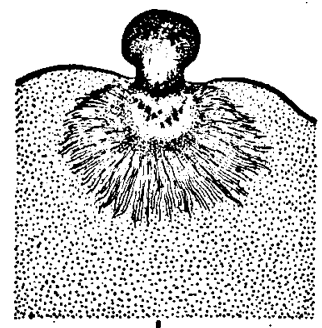

b.

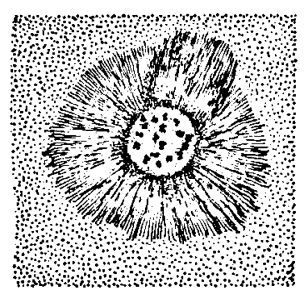

e.

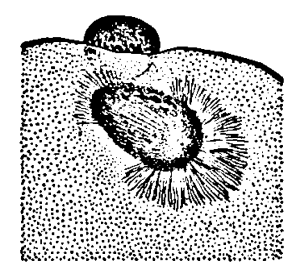

h.

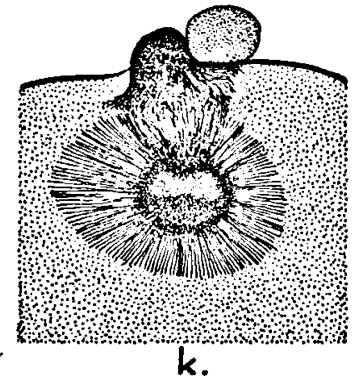

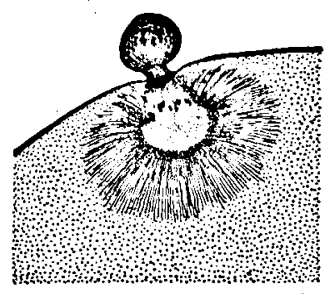

c.

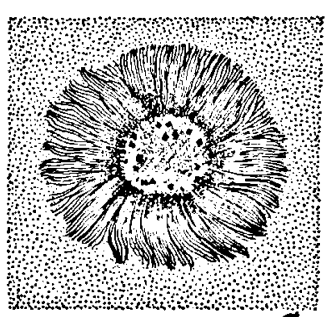

f.
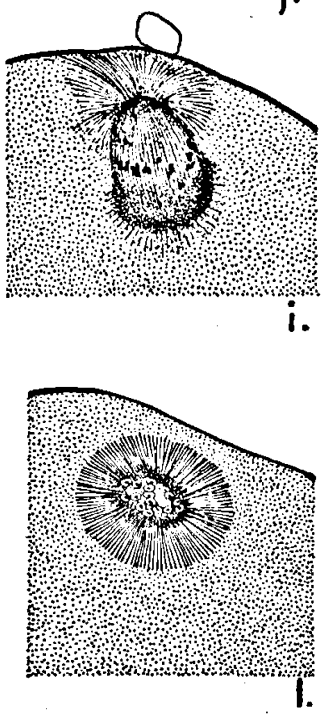

Fig. 2. From first to second maturation division. Eggs in distilled water. $a$. Telophase of first maturation division. $b-c$. Extrusion of first polar body. $d-f$. Grouping of dyads at surface of central body. $g$ - $\boldsymbol{h}$. Reduction of astral rays. Transformation of central body into spindle. i. "Acorn"-stage of $2^{d}$ maturation spindle. Rotation of spindle. Formation of new aster at its outer end. $j$. Metaphase stage of $2^{d}$ maturation spindle. Spermaster fused with its inner end. $k$. Telophase of $2^{d}$ maturation division. $l$. Beginning swelling of chromosomes into karyomeres. 
more reticular arrangement. However, the structure of the "central body" (I will use this term here for the sake of brevity) does not agree either with that of the cytoplasm surrounding the asters; apart from the fact that it is free from inclusions (yolk spheres, mitochondria), it has a denser appearance in the sections and a greater basophily. Around the margin, where the "central body" passes into the zone of astral rays, there is a definite circle of microsomes staining deeply with iron haematoxylin, which are lying between the basal parts of the astral rays.

The fibers of the central spindle between the two groups of dyads are somewhat thickened in the region of the spindle equator, forming in this way a transverse band which has a striped appearance ("cell plate") (fig. Ic, 2a). The same phenomenon has been observed during cleavage in Limnaea (RAven 1946). With further extrusion of the polar body this band comes to lie in a line with the egg surface; it is in this region that the boundary between egg and polar body is formed (fig. $2 b, c$ ).

The first polar body temporarily has the shape of a toad-stool; it is pinched off by a progressive narrowing of the stalk connecting it 'with the egg (fig. $2 b, c)$. Thereby the remnant of the maturation spindle in the region of this stalk is compressed. The "cell plate" mentioned above now stains heavily with iron haematoxylin (fig. 2b). With further constriction, this staining extends to the rest of the stalk (fig. 2c) which finally is transformed as a whole into a dark-stained "mid-body". This may be found for some time at the surface of the egg beneath the first polar body (till about the stage of fig. $2 \mathrm{~h}$ ), but then it is resorbed.

At the end of anaphase of the first maturation division the central group of dyads reaches the margin of the "central body" of the deep maturation aster (fig. 2a-c). Here their movement stops; they do not invade the central body, but remain at its surface or in its outer part (in tangential sections of the egg, like those of fig. $2 \mathrm{e}$ and $\mathrm{f}$, they may appear to be lying in the central body; actually, however, they are situated at a higher level). Their double nature is clearly visible; each dyad consists of two short rods which are closely applied. In favorable sections which contain all dyads, like those of fig. $2 e$ and $f$, it is evident that $I 8$ dyads are present; hence, this must be the haploid number of chromosomes in Limnaea stagnalis.

When the dyads first reach the central body, they form a tightly-packed heap (fig. 2a, d). Soon, however, they begin to separate, moving along the outer surface of the central body until they are grouped in a more or less irregular ring (fig. 2f). At the same time, the central body itself is increasing in size (fig. 2a-f). Its shape is that of a rotation ellipsoid, somewhat flattened parallel to the egg surface. The dimensions of the central body, which are about $12.8 \times 12.0 \mu$ at the stage of fig. $2 \mathrm{a}$, have increased to, on an average, $16.8 \times 15.2 \mu$ at the stage of fig. 2e. At the same time, the aster is slowly decreasing in size from $42 \times 3^{8 \mu}$ to $35 \times 3^{\circ} \mu$. (In the drawings of fig. 2 , 
which represent single sections, this is not obvious). At first, the astral radiations remain clearly visible. At a certain moment, however, they begin to become blurred and rapidly decrease in length. At the same time, the central body begins to elongate in a transverse or oblique direction (fig. 2 g). It becomes more or less egg-shaped, one end being pointed and the other blunt. When it has an oblique position, its pointed extremity is always directed towands the surface. Now in this half a longitudinal striation appears, indicating a parallel orientation of the cytoplasmic micellae (fig. $2 \mathrm{~h}$ ). The other half, which widens still more and is surrounded by a broad zone of microsomes staining deeply with iron haematoxylin, at first retains its original appearance; the whole structure, at this moment, has the shape of an acorn in its cup (fig. 2i). The dyads, which are at first arranged more or less at random, now concentrate in the middle region of this structure, where they form an irregular ring. The longitudinal striation extends more and more into the other half; in this way, the "central body" is transformed into the second maturation spindle, which places itself at right angles to the surface (fig. $2 \mathrm{~h}-\mathrm{j}$ ).

The original radiations of the first maturation aster meanwhile have nearly entirely disappeared. At the outer end of the developing second maturation spindle, however, a new aster appears (fig. h-i). The deep end of the spindle, on the contrary, is surrounded by an indistinct fringe of disappearing astral rays only. However, at the same moment that the second maturation spindle has attained its definite structure and position, with the dyads arranged in the equatorial plate, its deep end is provided with a big and well-developed aster, containing in its centre a large clear "central body" (fig. 2j).

This sudden appearance of an aster, at the height of its development, at first seemed quite surprising, until it was proved that it is the spermaster which fuses secondarily with the deep end of the maturation spindle. Unexpected as this may seem at first, a complete series of stages proves beyond all doubt that this is the case:

BRETSCHNEIDER (1948) showed that all but one of the spermia entering the Limnaea egg at insemination are dissolved in the cytoplasm. The fertilizing sperm at first undergoes no changes worth mentioning. In freshly-laid eggs, the sperm head was found as a compact dark body, often lying in a vacuole, immediately beneath the egg cortex (RAven 1945). The whole spermia, including the middle piece and the tail, enter the egg; in some cases, in freshlylaid eggs the sperm head is still attached to the tail, but soon it breaks away from it.

The spermaster makes its appearance during the telophase stage of the first maturation division. At first, it is a very small structure, measuring about Io $\mu$ in diameter only. It has no "central body", the astral rays meeting in one point at the centre of the spermaster (fig. Ic). Its position varies; it may lie near the centre of the egg or shifted towards one side, but never quite near to the egg surface. That it really is a spermaster is proved by the 
fact that in a few instances it has been found to be situated at the end of a disintegrating piece of sperm tail (cf. fig. Id). During the transformation of the central body of the first maturation aster into the second maturation spindle, the spermaster grows rapidly in size, while a distinct "central body" appears in its centre. At the same time, it shifts its position towards the animal pole, approaching, in this way, the deep end of the developing second maturation spindle (fig. Id). At the moment this spindle has finished its rotation with respect to the surface and the dyads have arranged themselves into an equatorial plate, the spermaster joins its deep end and forms, in th:s way, the deep aster of the second maturation amphiaster (fig. $2 \mathrm{j}, \mathrm{re} 1$ )).

The further history of this amphiaster offers no peculiarities; fig. Ie shows an anaphase stage, fig. $2 k$ an early telophase stage of the second maturation division. When the second polar body is pinched of $f$, a distinct "mid-body" is formed (fig. If). Immediately afterwards, the chromosomes which have reached the periphery of the "central body" of the deep aster, begin to swell into little vesicles (fig. 21). This swelling continues until these karyomeres have grown to rather big nucleus-like bodies. Meanwile, they leave their position at the boundary of the "central body" and assemble immediately beneath the egg cortex at the animal pole (fig. Ig). The remaining aster (which is the former spermaster) shifts to a deeper position near the centre of the egg. Its central body has grown very big and forms an area of clear, highly vacuolated protoplasm, which is surrounded by a denser zone, in which at first the astral rays are still clearly visible; later, they become more and more inconspicuous (fig. Ig).

The sperm nucleus has retained its subcortical position and compact structure till the end of the second maturation division. At telophase of this division, when the chromosomes have reached the "central body" of the deep aster and begin to swell into karyomeres, suddenly the sperm nucleus begins to move towards the animal pole. At the same time, it swells and develops into a male pronucleus. In a number of cases this is found for some time near the centre of the egg, immediately beside the spermaster (fig. Ih) or in its central clear area (fig. Ig). Often, however, it shifts towards the animal pole at once, which it may have reached already 20 minutes after the extrusion of the second polar body. The male pronucleus, when it has reached the animal pole, comes to lie immediately beneath the egg karyomeres; somewhat later, the latter unite progressively to the female pronucleus (fig. Ii).

\section{DISCUSSION}

Two points in the series of events described above deserve a special consideration: $I^{\circ}$ the formation of the second maturation spindle by a direct transfor-

I) The series of drawings of fig. I, which have been published in my paper of 1945 , already suggest this succession of events. However, at that moment I did not dispose of enough material to prove it beyond doubt. 
mation of the inner central body of the first maturation amphiaster; $2^{\circ}$ the temporary fusion of the spermaster with the deep end of the second maturation spindle.

I. In the literature, exact descriptions of the formation of the second maturation spindle are relatively rare. Text books of embryology, as a rule, only contain statements to the effect that the chromatic parts of the nucleus af ter the completion of the first maturation division immediately arrange themselves into an equatorial plate of a new spindle, the second maturation spindle. As to special investigations on maturation and fertilization, detailed descriptions of this process are found especially in the older literature. Generally, the formation of the second maturation spindle is described in the following way: The central body ("centrosome" of some authors, "centrosphere" of other ones) of the deep aster of the first maturation spindle contains in its centre a deeply staining granule, called "centriole" or "centrosome" "by different authors. Already before the completion of the first maturation division, this granule divides and the two daughter granules move apart. They become the focal points of new astral radiations, while a central spindle is formed between them. In this way, a new amphiaster, consisting of a central spindle with two asters, arises in the area of the old "centrosphere". The chromosomes of the first maturation division now come secondarily into contact with this spindle and arrange themselves into the equatorial plate. In this way, the process is e.g. described by ByRnes (1900) in Limax agrestis and by LINVILLE (1900) in Limax maximus.

Somewhat different is the description given by Von Kostanecki \& WierZEJSKI (1896) in Physa fontinalis. They do not pay much attention to the "centrosphere"; according to their description, the inner "centrosome" of the first maturation spindle divides and the two halves move apart, giving rise to the formation of the central spindle.

According to Garnault ( 1889 ), in Helix aspersa and Arion empiricorum after the formation of the first polar body two cases may occur: either a vesicular nucleus is formed by the chromosomes gathering in the central area of the aster, which has increased gradually in size, and a membrane is formed at the limit between sphere and aster; or, the chromosomes remain in the superficial parts of the egg, while the "sphère attractive", after disappearance of the first maturation spindle, directly forms a second spindle with two asters, which comes into relation with the chromosomes only secondarily. It is evident that the author has not succeeded to arrive at a consistent interpretation of the observed facts.

In comparing the descriptions and the accompanying drawings of the abovementioned authors, all relating to the eggs of the Pulmonates, it is striking that a great variation exists, especially as regards the composition of the central body. V. KostANECKI \& WIERZEJSKI describe and picture rather big granular "centrosomes", which stain a deep black with HeIDENHAIN's iron haematoxy- 
lin. In part of the cases a double "centrosome" is represented, either at one or at both ends of the first or second maturation spindle. The moment of "reduplication" of the "centrosome" varies much; single centrosomes are found as late as the telophase stage, double centrosomes as early as the metaphase stage of the maturation divisions. The size of the centrosome is also very different. In some cases it is surrounded by a circular clear area ("sphere"), in other instances this is lacking and the astral rays are continuous with the "centrosome"; sometimes, within the clear "sphere" the "centrosome" is surrounded by a somewhat darker area. These different appearances are attributed to different physiological "states of stimulation" of the cytoplasmic rays. Similar results were obtained as regards the "centrosomes" of the spermaster. Single and double "centrosomes" were found both in early and late phases of the fertilization process.

Quite different is the description of the central bodies of Limax agrestis by BYRnEs (1900). The "centrosphere" of the first maturation spindle has a distinct peripheral zone, staining intensely with iron haematoxylin. It is marked off distinctly from a perfectly clear structureless inner zone; sometimes, however, the concentric layers of the "centrosphere" are less sharply outlined. In other cases, the "centrosphere" is composed of four concentric rings, which are alternately dark and light; the astral rays diverge from the periphery of the outermost dark ring. Within the clear inner zone of the centrosphere there is a deeply staining central mass, consisting of several granules that form the "centrosomes"; they are often grouped irregularly. In haematoxylin the "centrosomes" stain almost black. Sometimes, the centres of the asters fail to show deeply staining central granules. After the extrusion of the first polar body the "centrosphere" undergoes a series of changes. The zones become less sharply defined as the sphere continues to enlarge. The astral rays begin to shorten. When the centrosphere reaches its maximum size it becomes uniformly very finely reticular and loses its strong affinity for staining reagents. In the centre of the sphere the centrosomes now are often distinguishable as two tiny, deeply staining granules; sometimes, however, no centrosomes are visible:

Still another description is given by Linville (I900). In Limax maximus, the "centrospheres" of the first maturation spindle are composed of a central, pale, reticulated area, and a very thick wall. No "centrosome" was found in specimens fixed by different methods and stained with HeIdenhain's iron haematoxylin for varying lengths of time. After the extrusion of the first polar body, the central reticulated area has increased in volume, and two centrosomes have made their appearance as extremely small granules. In Limnaea elodes, the centrosome of the first maturation spindle sometimes appears stained faintly, sometimes very deeply. When deeply stained, it varies in size from an extremely small body to one of the size of the centrosphere itself; in other cases, at the centre of the aster appears a mass of minute granules. After the first polar body has been formed the inner centro- 
sphere is composed of two parts, a very small, central clear area, of spherical form, and a very much larger non-spherical enveloping structure; at the exact centre of the inner part, there is a minute centrosome, but with no indication of division. At a slightly later stage, the centrosphere shows a decided increase in size. Only in a single case, a very early stage in the formation of the first maturation spindle, two centrosomes were found. The author is unable to say what relation the enlarging centrosphere bears to the formation of the second maturation spindle, but considers the possibility that the latter is formed de novo within the still persisting centrosphere.

My own observations in Limnaea stagnalis have shown the following facts: $I^{\circ}$ the "central bodies" of the first maturation amphiaster have the form of rather big clear cytoplasmic areas, which differ, apparently, in their submicroscopic structure from the surrounding region of astral rays; their appearance and colouring in the sections may vary somewhat according to fixation and staining; $2^{\circ}$ though occasionally small granules may be found in the central body, their occurrence, number and localization are very variable; in no case true "centrioles" have been observed, although most of the eggs had been stained with HeIdenhain's iron haematoxylin, which is considered one of the best staining methods for demonstrating these bodies; $3^{\circ}$ after the extrusion of the first polar body, the inner central body increases in size and transforms as such into the second maturation spindle. The asters of this second maturation amphiaster do not arise within the central body; the outer one develops from astral radiations appearing in the cytoplasm outside the original central body, the inner aster is supplied by the spermaster, which fuses secondarily with the inner end of the second maturation spindle.

In view of the great diversity in the observations relating to the central bodies in Pulmonate eggs (but also in other cells), one is reminded of the critique of the usual concepts concerning these structures which has been given by FRY (I929-1933). In a series of papers this author showed that the appearance of the central bodies (e.g. in Echinarachnius and Chaetopterus eggs) is dependent, not only on the stage of development, but also on the method of fixation and the temperature at which the eggs had been kept. From these observations he draws the conclusion that the so-called central bodies are nothing but coagulation artifacts of the focal points of astral rays, and that the supposed "centrioles" are actually cytoplasmic granules that have a random occurrence at the mid-point of asters.

As regards the "central body" as such, in my opinion this conclusion of $F_{R Y}$ is going somewhat too far. When he declares: "There are no central bodies" (1929d, p. 4), this is at variance with his own observation that the central areas of live asters are perfectly hyaline, whereas a radial configuration is distinctly visible in the outer parts (1929a, p. 124). Both from this observation and from the fact that in sections in most cases the central areas of the asters differ considerably in structure from the outer parts, it may be con- 
cluded that, except in very young asters, the central area is characterized by a submicroscopic structure which differs from that of the region of astral rays. This central area may be called a "central body", on the understanding that this term does not imply its continuity throughout the life of the cell as a separate entity.

As concerns the "centrioles", however, the conflicting descriptions of these bodies in Pulmonate eggs suggest that $F_{R Y}$ is right in his critical attitude. Presumably, often coagulation products in the central area of the asters or cytoplasmic granules accidentally present in this region have been misinterpreted as "centrioles". As FRY rightly remarks, most investigators select certain of the observed sections as "normal", whereas the others are dismissed as "poorly fixed". This method is open to the danger that the "normal" course of events is determined upon the basis of incomplete and partial data, and that, moreover, preconceived opinions in a large measure falsify the conclusion drawn from such studies. The investigator is tempted to lay undue stress on any picture fitting into a more or less diagrammatic representation of the course of events, and to dismiss all those cases that are conflicting with it. It is to be remembered that any structure that shows wide diversity as to form, location, physical appearance, or number, must be viewed with great scepticism. Such is the case with the so-called "centrioles" in the eggs of the Pulmonates, and perhaps in many other eggs as well. These considerations do not mean that the processes leading to the formation of the second maturation amphiaster in Limnaea stagnalis are alleged "typical" for the eggs of Pulmonates. It is possible that in other members of this group the course of events is somewhat.different. However, our observations form a warning against a too schematical representation of this phase of development; only an unprejudiced study of the processes involved on a sufficiently great material can give us a true knowledge of the relationship between the various structures of the egg cell.

2. As far as I know, in no case a temporary fusion of the spermaster with the inner end of the second maturation spindle, as observed in Limnaea stagnalis, has been described up till now. According to the usual representation, the spermaster, which arises from a centre situated in the middle piece of the spermium, migrates, either before or after the completion of the maturation divisions, towards the centre of the egg or to the region of the animal pole. Already during its migration, it may divide and form a small amphiaster (e.g. in Physa according to v. Kostanecki \& WierzejSki I896); in other cases it remains single, however. In most cases, the sperm nucleus accompanies the spermaster in its migration; in other instances, however, as in the Pulmonates, the migration of both structures takes place independently. The inner aster of the second maturation amphiaster may remain visible for some time after the extrusion of the second polar body, but ultimately it disappears in all cases. 
The spermaster may also disappear (e.g. Linville 1900: Limax and Limnaea), or it may directly transform into the amphiaster of first cleavage.

Very complicated is the evolution of the spermaster in Limnaea stagnalis appressa Say, as described by CRABB (1927). The early spermaster occurs in ova which have not undergone the first maturation division; its size equals that of the early egg asters. This primitive spermaster, or archiaster, is characterized by having long stout rays and "indifferently formed" centrosomes. Presumably, these sperm archiasters reach their maximum size during the prophase of the first maturation division. They normally undergo a sort of metamorphosis; during this period there is a marked reduction in the length of the rays and a great condensation of chromophilic substance in the centrosome. Subsequently, the typical aster reforms from the granules of the disintegrated centrosome of the archiaster and reappears as a very small aster having short delicate rays and a very condensed deep-staining centrosome. The amphiaster most commonly arises from this aster, about the time of the first maturation of the egg, but it may arise earlier.

Contrary to this, in Limnaea stagnalis L: I never found a spermaster before the telophase stage of the first maturation division; it is not preceded by an "archiaster", nor does it transform into an amphiaster. After its temporary fusion with the second maturation spindle, it is lying for some time near the centre of the egg or between this and the animal pole, then it vanishes completely. No visible relation exists between the spermaster and the cleavage amphiaster which appears a short time before first cleavage.

It may be that the behaviour of the spermaster in Limnaea stagnalis stands quite alone. On the other hand, in view of the above-mentioned considerations it appears possible that too often the evolution of the spermaster has been deduced from an insufficient material, taking into account only selected sections which fitted in with a preconceived idea as to its fate. Hence, it is possible that future investigations will show that also in the eggs of other species a fusion of the spermaster with the second maturation spindle occurs.

\section{SUMMARY}

r. The maturation divisions of the egg of Limnaea stagnalis L. have been studied.

2. The haploid number of chromosomes is 18 .

3. At the telophase of the first maturation division, the central group of dyads reaches the surface of the inner central body of the first maturation amphiaster.

4. This central body directly transforms into the second maturation spindle.

5. The aster surrounding this central body gradually disappears. New astral radiations are formed in the cytoplasm near the outer end of the newly formed second maturation spindle. 
6. The spermaster, which appears during the telophase of the first maturation division, fuses secondarily with the inner end of the second maturation spindle and forms the inner aster of the second maturation amphiaster.

7. After the extrusion of the second polar body, this aster shifts to a deeper position near the centre of the egg, where it remains visible for some time, but ultimately disappears.

8. The egg chromosomes swell into karyomeres immediately after the extrusion of the second polar body. At the same time, the sperm nucleus begins its migration towards the animal pole.

\section{LITERATURE}

Bretschneider, L. H., 1948. Insemination in Limnaea stagnalis L. Proc. Kon. Ned. Akad. v. Wet. Amsterdam $51,358$.

Byrnes, E. F., 1900. The maturation and fertilization of the egg of Limax agrestis (Linné). J. Morph. 16, 201.

CRABB, E. D., 1927. The fertilization process in the snail, Lymnaea stagnalis appressa Say. Biol. Bull. $53,67$.

FRY, H. J., I929a. The so-called central bodies in fertilized Echinarachnius eggs. I. The relationship between central bodies and astral structure as modified by various mitotic phases. Biol. Bull. 56, IOI.

-, 1929b. - II. The relationship between central bodies and astral structure as modified by various fixations. Biol. Bull. 57, I3I.

- 1929c. - III. The relationship between central bodies and astral structure as modified by temperature. Biol. Bull. 57, 15 I.

- 1929d. A critique of the usual concepts concerning the mitotic mechanisms of the echinoderm egg. The Collecting Net 4, no. 6.

- 1932. Studies of the mitotic figure. I. Chaetopterus: central body structure at metaphase, first cleavage, after picro-acetic fixation. Biol. Bull. 63, I49.

-, 1933. - III. Chaetopterus: central body structure at metaphase, first cleavage, after using diluted fixatives. Biol. Bull. 65, 207.

Garnault, P., I889. Sur les phénomènes de la fécondation chez l'Helix aspersa et l'Arion empiricorum. Zool. Anz. I2, 10.

Kostanecki, K. v. \& A. WierzejSki, i896. Uber das Verhalten der sogen. achromatischen Substanzen im befruchteten Ei. Nach Beobachtungen an Physa fontinalis. Arch. Mikr. Anat. 47, 309.

Linville, H. R., 1900. Maturation and fertilization in Pulmonate Gasteropods. Bull. Mus. Comp. Zool. Harvard Coll. 35, 213.

Raven, Chr. P., 1945. The development of the egg of Linnaea stagnalis L. from oviposition till first cleavage. Arch. Néerl. Zool. 7, 91.

- I946. The development of the egg of Limnaea stagnalis L. from the first cleavage till the trochophore stage, with special reference to its "chemical embryology". Arch. Néerl. Zool. 7, 353. 